
la Grande Guerre

\title{
Sur les traces de la Grande Guerre dans le paysage en Lorraine centrale au Nord-Est de Nancy (Moselle, Meurthe-et-Moselle)
}

Dominique Harmand, Denis Mathis et Emmanuel Chiffre

\section{OpenEdition}

\section{Journals}

Édition électronique

URL : https://journals.openedition.org/geohist/612

DOI : 10.4000/geohist.612

ISSN : 2264-2617

Éditeur

Association française de la Revue de géographie historique

Référence électronique

Dominique Harmand, Denis Mathis et Emmanuel Chiffre, «Sur les traces de la Grande Guerre dans le paysage en Lorraine centrale au Nord-Est de Nancy (Moselle, Meurthe-et-Moselle) », Revue de géographie historique [En ligne], 14-15 | 2019, mis en ligne le 28 mai 2018, consulté le 12 juin 2021. URL : http://journals.openedition.org/geohist/612 ; DOI : https://doi.org/10.4000/geohist.612

Ce document a été généré automatiquement le 12 juin 2021.

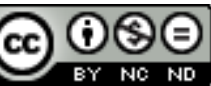

$\mathrm{Ce}(\mathrm{tte})$ œuvre est mise à disposition selon les termes de la Licence Creative Commons Attribution -

Pas d'Utilisation Commerciale - Pas de Modification 4.0 International. 


\section{Sur les traces de la Grande Guerre dans le paysage en Lorraine centrale au Nord-Est de Nancy (Moselle, Meurthe-et-Moselle)}

Dominique Harmand, Denis Mathis et Emmanuel Chiffre

\section{Introduction}

1 L'objectif de cette contribution est double. Il s'agit tout d'abord de décrire précisément le cadre morphostructural des trois batailles de Morhange, de la Trouée de Charmes et du Grand-Couronné, complétant le chapitre de l'ouvrage La Terre et le Fu, publié par l'Association des Géologues du Bassin de Paris (Bergerat (dir.), 2018). Il s'agit ensuite d'exhumer les vestiges de la Grande Guerre dans l'Empire allemand, dans un secteur appartenant au même cadre morphologique où la frontière est restée à peu près stable pendant les 4 années du conflit. Il sera nécessaire de replacer enfin ces vestiges dans le cadre des diverses positions militaires dans la Lorraine centrale entre le Grand Couronné et la Seille d'une part et les Vosges gréseuses de l'autre.

Pour cet article, nous avons d'abord mis à profit les nouveaux travaux en géologie et en géomorphologie acquis depuis une trentaine d'années (Le Roux, 1978, 1999; Gély et Hanot (dir.), 2014 ;Le Roux et Harmand, 2014, 2015 ; Harmand et al., 2018). Nous avons ensuite cartographié, à l'aide d'un GPS, les vestiges reconnus dans la forêt de Grémecey, sur le plateau et sur les versants du vallon Vassieux, puis placé ceux-ci sur un MNT à l'aide du logiciel ArcGIS (Esri). La cartographie réalisée admettra une imprécision de l'ordre de $5 \mathrm{~m}$. Enfin, nous avons interrogé la mémoire collective des villages situés à proximité du massif forestier. 


\section{Le cadre morphostructural des trois batailles de Morhange, de la Trouée de Charmes et du Grand- Couronné}

Dès les premières semaines du conflit, du 10 août au 13 septembre 1914 (Cochet, 2014), les trois offensives, française à Morhange et allemandes de la Trouée de Charmes et du Grand Couronné de Nancy, toutes trois suivies de contre-offensives, assistent à une stabilisation du front àpartir de la fin de l'année 1914(Doc. 1).

Document 1 : Carte géologique simplifiée des sites des batailles de Morhange, de la trouée de Charmes et du Grand Couronné.

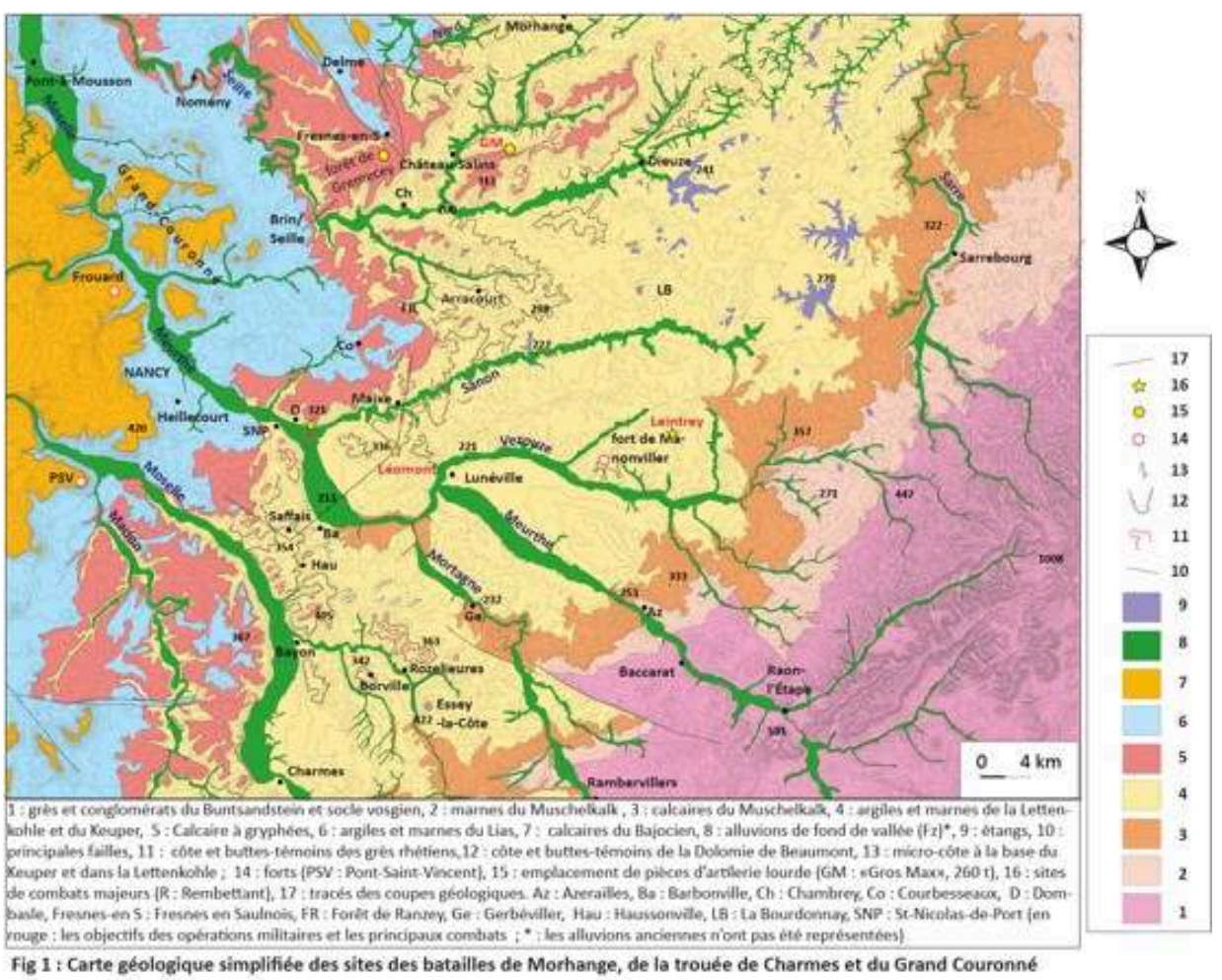

Dès lors, il semble pertinent de montrer, d'une part, quel rôle a joué le relief de côte pendant les 4 années du conflit dans un secteur où le relief est peu marqué ; et d'autre part, quelles traces jalonnent les anciennes positions militaires, notamment en Moselle sur les marges sud-ouest de l'Empire allemand. La forêt de Grémecey, située à l'ouest de Château-Salins, est privilégiée dans cette étude.

Les trois grandes batailles d'août et de septembre 1914 se déroulent pour l'essentiel d'entre elles dans la Lorraine centrale, région qui dépasse rarement $300 \mathrm{~m}$ d'altitude, entourée par deux régions plus élevées, nommées respectivement Lorraine orientale et Lorraine occidentale (Liedtke et al.,2010). La Lorraine orientale localisée au sud-est d'un axe Sarrebourg-Rambervillers, coïncide avec les reliefs des Vosges gréseuses armés par les grès et conglomérats du Buntsandstein où les altitudes atteignent $1008 \mathrm{~m}$ au Donon (Durand, 2014 ; doc. 1). La Lorraine occidentale, située au nord-ouest de Nancy, correspond au relief de côte le plus marqué, dépassant $400 \mathrm{~m}$ au sommet des côtes. Lacôte de Moselle, précédée au nord-est de Nancy par les buttes-témoins du Grand Couronné limite à l'ouest la Lorraine centrale. 
Dans celle-ci, les côtes sont moins perceptibles pour un œil non averti. C'est le cas àl'est, pour la côte de Lorraine (ou côte des calcaires du Muschelkalk) dont les altitudes sont maximales au droit des percées cataclinales de la Sarre à Sarrebourg (322 $\mathrm{m})$ de la Vezouze $(357 \mathrm{~m})$ ou de la Meurthe $(333 \mathrm{~m})$ à Azerailles. Toutefois, la complexité est beaucoup plus grande pour les côtes de l'Infra-Lias situées à l'ouest de la région étudiée. Cette complexité est due tout d'abord à des facteurs structuraux, la Lorraine centrale correspondant dans une large mesure avec les "dômes et cuvettes du Xaintois-Lunévillois» (Le Roux, 1999), faisant affleurer à la même altitude des formations d'âge différent (Doc 2). La complexité est due ensuite à la dissection poussée dans les formations du substratum, en majorité peu résistantes à l'érosion: Marnes irisées du Keuper et marnes du Lias, et à l'opposé, la faible épaisseur des formations les "plus résistantes »: Dolomie de Beaumont (Keuper), grès rhétiens (Trias) et Calcaires à gryphées de l'Hettangien-Sinémurien (Lias), est favorable à une dissection poussée du relief de côte réduit en buttes-témoins, comme entre Château-Salins et Dieuze, ou àl'estde Bayon (Fig. 1 et 2).

\section{Document 2 : Coupes géologiques des côtes de Saffais.}

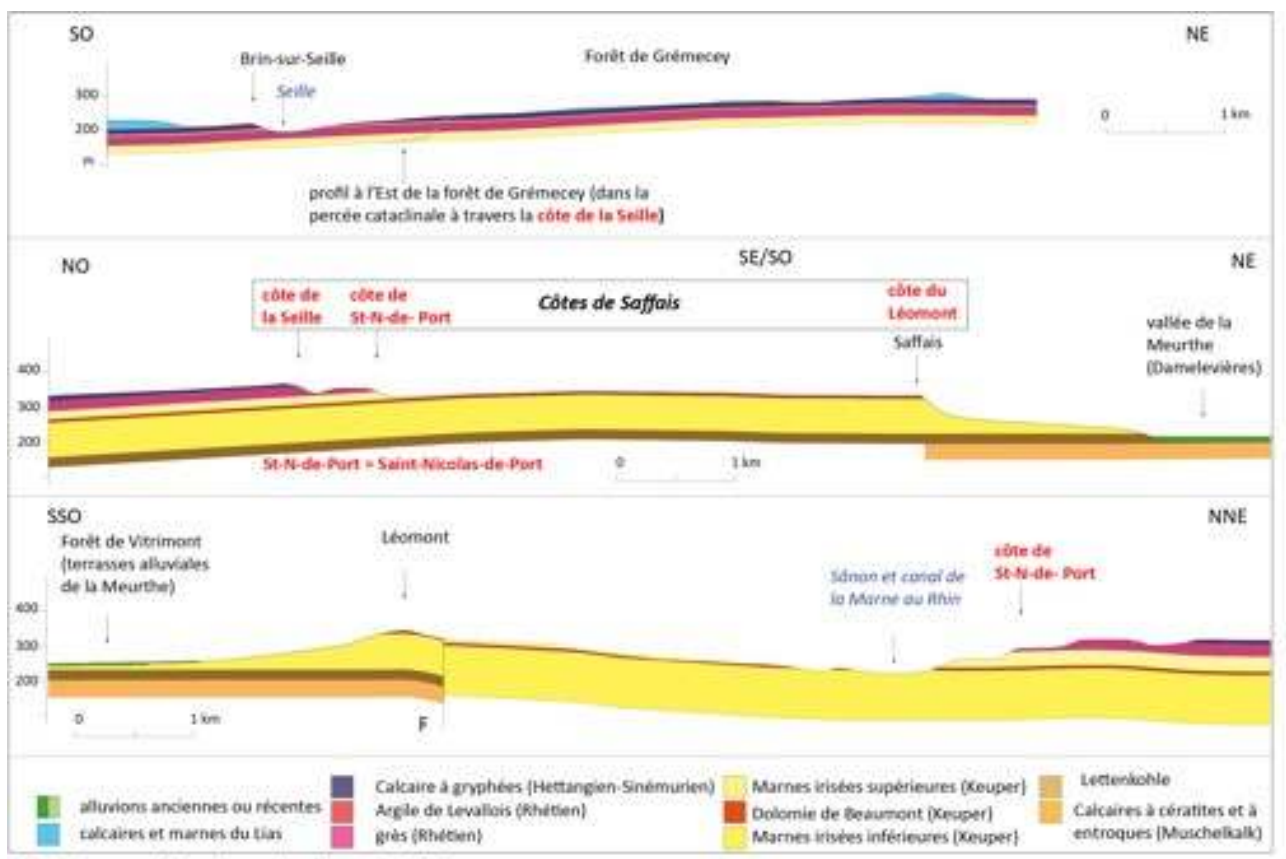

Fig 2 : Coupes géologiques des côtes de Saftais

7 En fait, la pluralité des côtes de l'Infra-Lias a été soulignée par Schmitthenner (1923), géomorphologue allemand reconnu, qui a été affecté comme géologue de guerre dans le secteur de Verdun entre septembre 1915 et novembre 1918 (Deshaies, 1999). En effet, Schmitthenner nomme la " côte du Lias et du Rhétien» [Lias-Rhät Stufe], «côtes de Saffais » lesquelles dominent de près de $200 \mathrm{~m}$ la vallée de la Meurthe à Blainville-surl'Eau. Saffais est d'ailleurs la seule localité qui est utilisée par Schmitthenner pour désigner une côte de l'est du Bassin parisien. Cette côte double est en effet constituée au sommet par la côte des Calcaires à gryphées ou, lorsque cette formation a été érodée, par les grès rhétiens, et en contrebas par la côte de la Dolomie de Beaumont ; la puissance de ces trois formations étant respectivement d'une quinzaine de mètres, d'environ 20-25 m et de moins de $10 \mathrm{~m}$. Les côtes de Saffais correspondent ainsi à trois côtes qui seront désignées dans cet article par la côte de la Seille pour la côte de 
l'Hettangien-Sinémurien, la côte de (Saint-Nicolas-de-) Port pour celle du Rhétien, et la côte du Léomont pour celle de la Dolomie de Beaumont.

8 Cette côte, le plus souvent oubliée par les géomorphologues, a en effet joué un rôle majeur lors des offensives de 1914. Tout d'abord au Léomont, la butte-témoin de $336 \mathrm{~m}$ d'altitude, située à l'ouest de Lunéville, est conquise à 8 reprises par les armées belligérantes, ce qui souligne la valeur militaire du relief de côte (Villate, 1925). La côte du Léomont est également bien représentée par les nombreuses buttes-témoins situées au «fond de la Trouée de Charmes", au nord-est de la localité éponyme. C'est notamment sur la butte-témoin de Borville que fut stoppée l'offensive allemande. Signalons également que le fort d'arrêt de Manonviller, localisé au nord de la vallée de la Vezouze, se trouve sur cette même côte, ce qui permettait d'avoir une vue imprenable sur la dépression de la Lorraine centrale et sur celle du Keuper.

Quant aux côtes de Port et de la Seille, elles jouèrent un rôle majeur lors des batailles de Morhange et du Grand Couronné, ainsi que lors de celle de la Trouée de Charmes où se trouvent des buttes-témoins de grès rhétiens.

\section{Les vestiges allemands de la forêt de Grémecey}

\section{A. Le contexte de la forêt de Gremecey}

10 Le secteur de la frontière de 1871, aujourd'hui fossilisée par le découpage départemental entre Moselle et Meurthe-et-Moselle, n'est pas, à proprement parler, une « frontière militaire » (Boulanger, 2002). En effet, elle n'a pas connu d'empilement par l'armée d'obstacles artificiels pouvant donner naissance à un front fortifié de campagne ou simplement un espace construit autour de pôles fortifiés. Ál'opposé, cette frontière a été partiellement démilitarisée par le déclassement des vieilles places fortes de Marsal ou de Phalsbourg (Mathis, 2016). Son marquage reste très symbolique (postes de douanes, gares monumentales et bornes frontières) mais souligne la rupture territoriale. Cette zone "d'entre-deux» doit être considérée comme une frontière « scientifique » (Boulanger, 2002), car elle tient compte d'avantages tactiques destinés à produire un territoire de rencontres et de bataille.

11 Dès l'automne 1914, malgré l'effort allemand vers la trouée de Charmes et les combats dans le secteur d'Avricourt, le front se stabilise. Du coté mosellan, il faut attendre l'année 1915 pour que la vie agricole cesse progressivement et qu'un territoire de guerre s'impose sur les terroirs agricoles mis en culture. L'évacuation des habitants au printemps 1915, permet aux armées de transformer les villages en points d'appui fortifiés intégrés à la première ligne de tranchées notamment sur la vallée de la Seille (Chambrey, etc.), ou en lieux de casernement en retrait des positions de combats. Ils sont progressivement intégrés au paysage de guerre et à ses ruines.

Actuellement, de nombreuses recherches effectuées notamment lors des commémorations du Centenaire ont exhumé un grand nombre de vestiges situés au sud de l'ancienne frontière, dans le département de Meurthe-et-Moselle (forêts de Champenoux, de Ranzey, de Bézange, etc.). En revanche, les vestiges situés en Moselle sont le plus souvent méconnus. Aussi était-il opportun de rechercher, dans un secteurtest, des polémo-paysages situés pendant la Grande guerre sur les marges méridionales de l'Empire allemand. Ce choix s'est porté sur la forêt de Grémecey qui se situe dans le même contexte morphologique que celui décrit dans la première partie de cet article. 
Le secteur cartographié se localise en effet sur le sommet de la côte de la Seille et sur les versants du ruisseau Vassieux, au nord de la percée cataclinale de la Seille à travers la côte éponyme. Or, l'existence d'une cuvette structurale à l'ouest de Brin-sur-Seille, au sud-ouest de la percée cataclinale, abaisse le plateau des Calcaires à gryphées dans la même direction (Le Roux, 1999), contraignant à renforcer les fortifications vers l'est en amont de cette percée. Situé sur les marges des 3 batailles de Morhange, de la Trouée de Charmes et du Grand Couronné, le secteur étudié se trouve au droit d'un front fixé sur la frontière de la Seille, qui n'a guère été modifié de 1914 à 1918.

\section{B. Les vestiges de la forêt de Grémecey :résultats}

14 Ces vestiges sont situés d'une part sur le plateau et consistent essentiellement en blockhaus en béton; d'autre part dans le vallon Vassieux où se trouvent surtout des restes de tranchées (doc. 7). Toutefois, ces deux types de traces se trouvent dans les deux contextes morphologiques. Dans ces deux secteurs, sous forêt, les arbres portent encore de nombreux vestiges de lignes téléphoniques repérables à leurs isolateurs - " potelets " (Fig. 9). La densité des lignes laisse deviner un important réseau de communication aérien qui couvrait tout le massif. De nombreux arbres portent 4 à 5 lignes de barbelés striant les troncs.

\section{Document 3 : Vestiges de la Grande guerre dans les 4 secteurs étudiés de la Forêt de Grémecey (D. Harmand).}

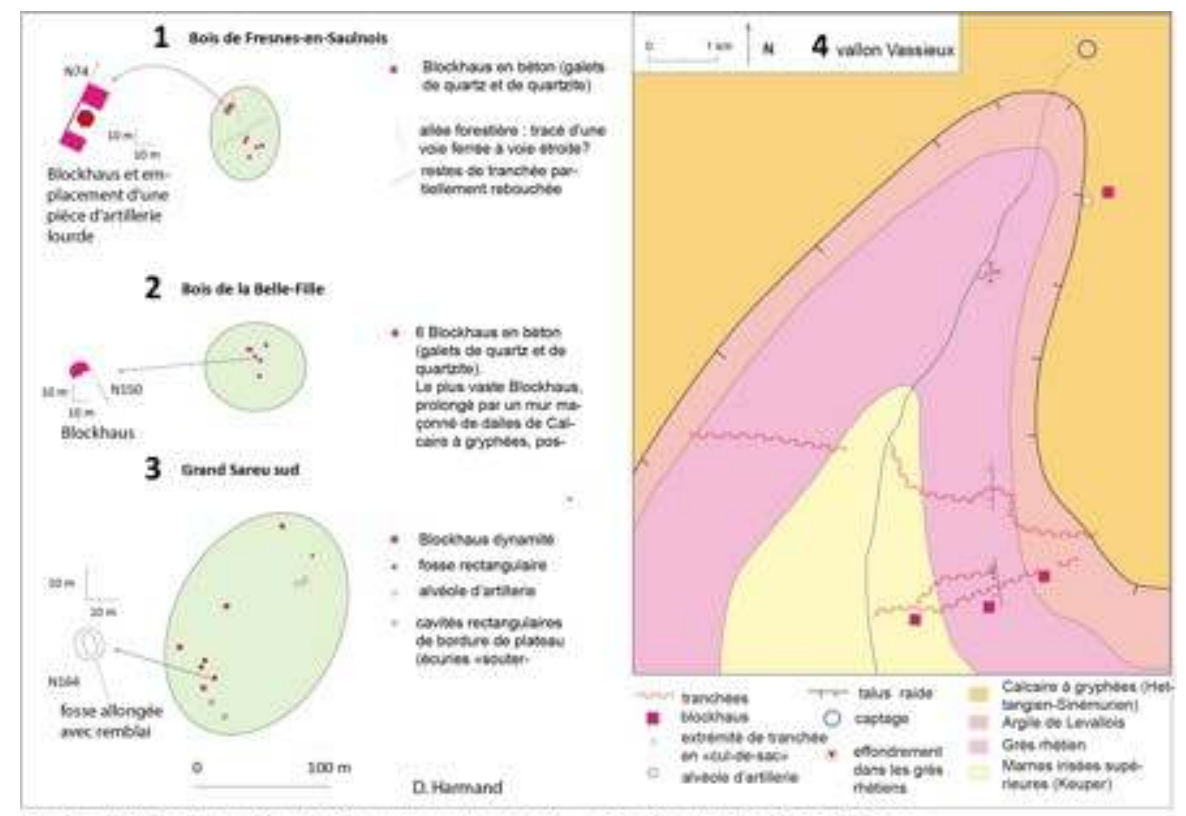

Fig. 7 : Vestiges de la Grande Guerre dans les 4 secteurs étudiés de la Forèt de Grèmecey

\section{a. Les vestiges sur le plateau}

Les vestiges observés et cartographiés au cours de cette étude se trouvent dans 3 secteurs : dans le Bois de Fresnes-en-Saulnois (secteur 1, doc. 7), dans le Bois de la Belle Fille (secteur 2) et au sud du Grand Sareu (secteur 3). Dans les deux premiers secteurs, les vestiges sont constitués essentiellement de blockhaus dispersés sur une superficie 
d'environ 12,5 ares. Dans le secteur 3, les vestiges, dispersés sur une surface d'environ 1,5 ha, sont constitués à la fois de blockhaus et de dépressions bordées d'un remblai.

Dans les secteurs 2 et 3, les blockhaus sont de dimensions modestes: le plus vaste observé dans le secteur 2 occupe une superficie d'environ $86 \mathrm{~m}^{2}$. Dans le secteur 1 (Bois de Fresnes-en-Saulnois), le plus éloigné du front, les blockhaus présentent deux originalités. Tout d'abord, deux d'entre eux sont plus volumineux et sont recouverts d'un remblai de plusieurs mètres de hauteur (doc. 4). Ils ont une superficie comprise entre 127 à $150 \mathrm{~m}^{2}$. Mais surtout, la plupart des blockhaus ont deux entrées, tandis que l'espace intérieur se prolonge par une cavité souterraine (non explorée à ce jour). Les matériaux, hormis deux murs maçonnés en dalles de Calcaire à gryphées observés dans le secteur 1 , sont constitués de béton formé renfermant des galets de quartz et de quartzite. Il est à noter que les murs sont dans un très bon état de conservation, un siècle après leur édification.

Document 4 : Un des deux blockhaus « jumeaux » de la forêt de Fresnes-en-Saulnois (secteur 1)

La pièce d'artillerie lourde se trouvait à droite sur la photographie (cl. D. Mathis)

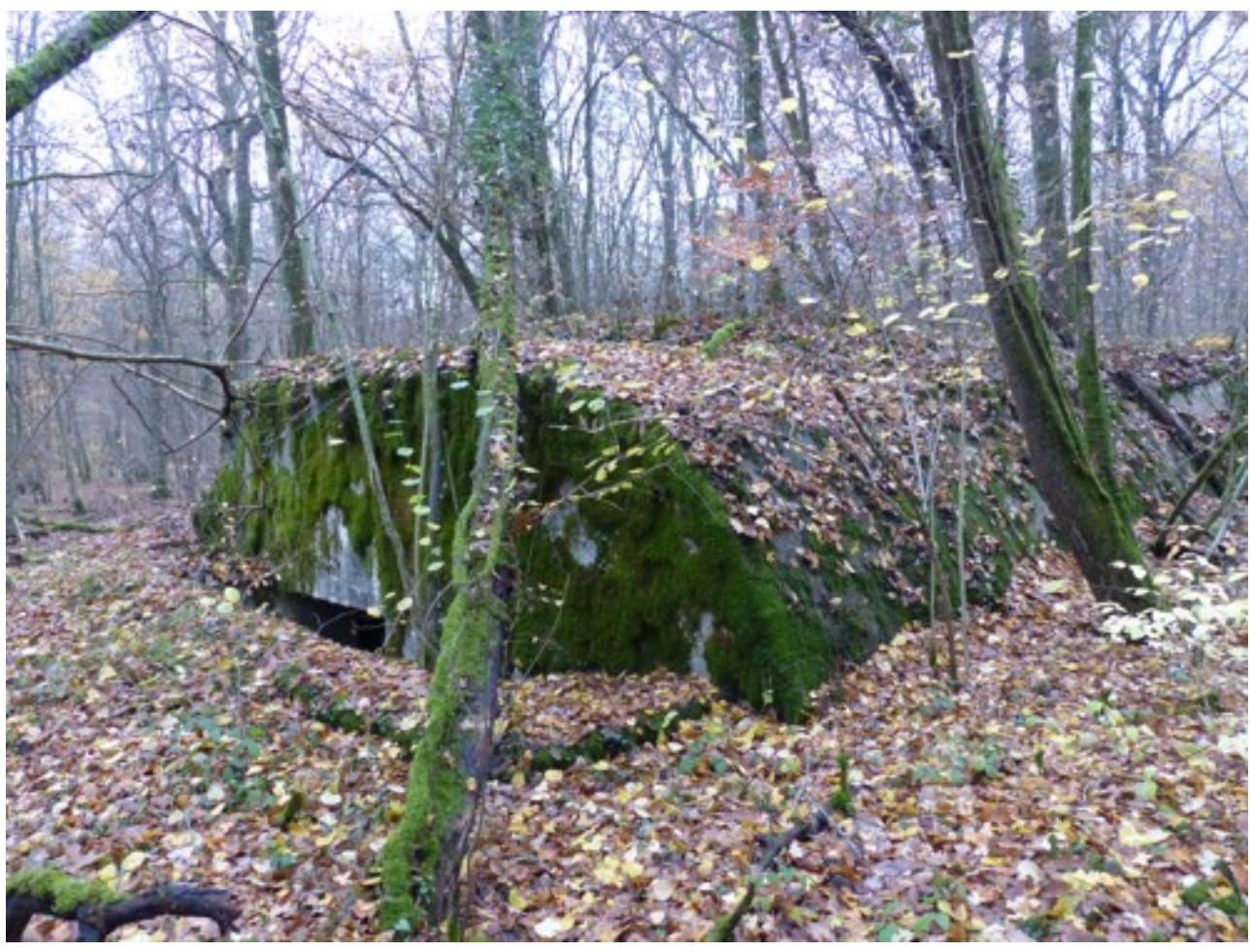

Les dépressions observées sur le plateau sont de trois types. On trouve d'une part plusieurs dépressions circulaires bordées d'un remblai assimilées à des alvéoles d'artillerie. D'autre part, on trouve plusieurs fosses rectangulaires elles-mêmes bordées d'un remblai (doc. 5). Enfin, en bordure du plateau, on trouve d'autresdépressions rectangulaires de dimensions pluri-métriquesalignées en bordure du chemin forestier (voir infra).

\section{Document 5 : Fosse rectangulaire à remblais de la Forêt de Grémecey}

(secteur 3, cl. D. Harmand) 


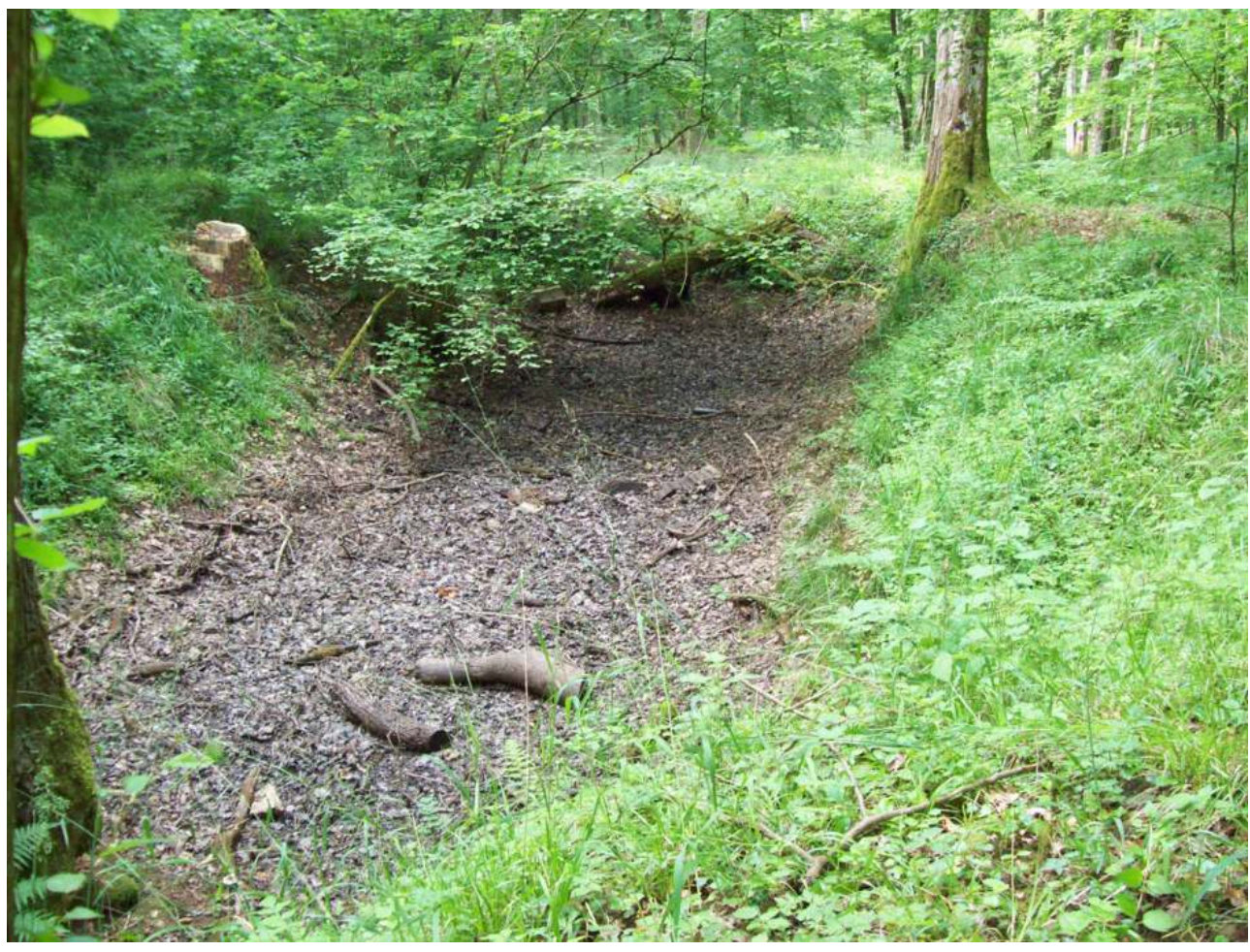

\section{b. Les vestiges dans le vallon Vassieux}

19 Dans le vallon Vassieux, plusieurs blockhaus situés sur le versant de rive gauche du ruisseau éponyme protégeaient les tranchées situées dans le vallon. Celles-ci, perpendiculaires à l'axe du vallon, constituaient plusieurs lignes de type "tranchées à traverses". Elles s'étendaient pour deux d'entre elles du rebord du plateau au ruisseau Vassieu, ce dernier permettant d'évacuer les eaux de pluie des tranchées, situées dans leur partie inférieure sur les Marnes irisées du Keuper, imperméables. Parfois, les tranchées, comblées, s'interrompent sur plusieurs mètres ou dizaines de mètres. De même, deux segments de tranchées observés s'interrompent brutalement au droit du talus modelé dans les grès rhétiens, ce dernier étant ponctué par des effondrements circulaires de dimensions métriques qui trahissent l'existence de cavités sous la surface du sol.

\section{Interprétations}

\section{a. Les vestiges apparents}

Les blockhaus appartiennent à deux types : la plupart, situés au sud du plateau et dans le vallon Vassieu sont des casemates-abris, à demi enterrées, implantées dès 1914 en milieu forestier comme l'attestent les nombreux vestiges de lignes téléphoniques dans les arbres. Le second type de blockhaus observé dans la forêt de Fresnes, au nord du secteur étudié, correspond à des soutes à munitions. De nombreux arguments vont dans ce sens : les 2 entrées - l'une pour les hommes, l'autre pour les munitions (Fig. 11) -, l'existence de galeries souterraines, le recouvrement de ceux-ci par des remblais, ainsi que l'éloignement de la ligne de front (7 à $8 \mathrm{~km}$ ). 

si leur profondeur initiale a été réduite par le colluvionnement. On peut émettre l'hypothèse que le découpage de la forêt en parcelles allongées de longueur ou de largeur obliques par rapport au vallon et appartenant à des propriétaires différents ne favorise pas une gestion raisonnée de la forêt, et a contribué à la fossilisation du polémo-paysage. Mais, il est probable que la longueur initiale des tranchées était probablement plus élevée, comme en Champagne et en Argonne (Taborelli et al.,2017). Notons également que la forêt de Grémecey a fait l'objet de combats en 1944, si bien que le paysage bouleversé par les quatre ans de guerre de la Première Guerre mondiale a pu être modifié pendant la Seconde.

Le dispositif des tranchées allemandes du vallon Vassieux met en évidence une adaptation au cadre géomorphologique. Il s'agissait, en effet, d'interdire l'accès au plateau des Calcaires à gryphées par un dispositif de tranchées orientées d'ouest en est et perpendiculaires à un vallon de direction subméridienne situé sur le front de la côte de la Seille. Or, ce dispositif est tout à fait comparable à celui du vallon du ruisseau des Fées situé à une dizaine de kilomètres plus au sud dans les lignes françaises, dans la Forêt de Ranzey, sur le front de la côte (doc 1). Le sentier de Ranzey, aménagé sous l'égide du conseil départemental de Meurthe-et-Moselle permet de découvrir la tranchée à traverses au tracé nord-sud, qui recoupait perpendiculairement un vallon de direction ouest-est. Le polémo-paysage est, ici, enrichi par des abris creusés dans les grès rhétiens (doc. 6) et un cantonnement «14/18» où il est possible d'observer l'emplacement des cuisines, la fontaine-lavoir aménagée en 1917, et le guéoir (C.D. Meurthe-et-Moselle, année inconnue).

Document 6 : Cavité creusée pendant la Grande guerre dans la forêt de Ranzey (Meurthe-etMoselle) dans les grès rhétiens

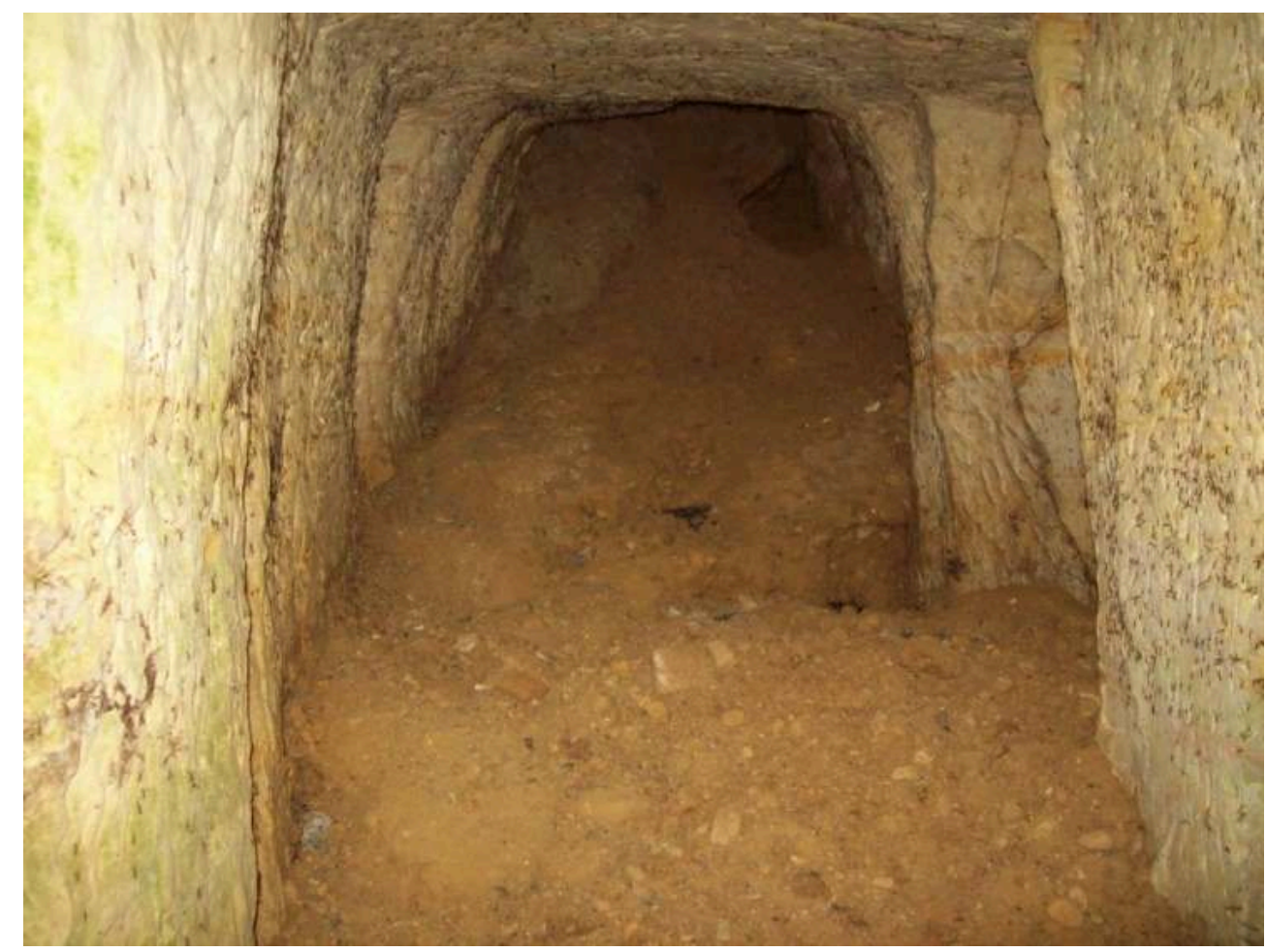




\section{b. Les vestiges fantômes (invisibles ou disparus)}

23 Le paléo-paysage de la Forêt de Ranzey situé dans les lignes françaises comporte, au nord du ruisseau des Fées, plusieurs abris creusés pendant la Grande Guerre dans les grès rhétiens. Ceux-ci affleurent sous forme d'un abrupt rocheux de 2 à $3 \mathrm{~m}$ de hauteur, exploité pour la pierre de taille au sud du vallon. Or, dans le vallon Vassieux, plusieurs indices semblent indiquer la présence de cavités dans les grès rhétiens, ainsi en est-il de l'interruption de 2 sections de tranchées sur le talus jalonnant les grès du sommet du Trias, et les désordres en surface liés à des effondrements sur cette même formation (Fig. 15). Aussi, nous pensons qu'il existe une galerie souterraine ou des abris creusés pendant la Grande guerre et qui ont été fossilisés ultérieurement par les formations de pente.

24 Concernant l'interprétation des fosses rectangulaires observées dans le secteur 3, on peut émettre deux hypothèses: soit elles étaient destinées à recevoir d'autres blockhaus qui n'auraient pas été construits, soit elles ont contenu des abris temporaires, détruits après la Grande guerre. Une comparaison avec les lignes françaises situées au sud de la Seille, dans la Forêt d'Amance, montre qu'il existait des casemates enterrées (Brin-sur-Seille, 2017) édifiées dès l'automne 1914. Ces casemates étaient constituées par une charpente en bois de chêne sur laquelle étaient posées des planches, le tout étant recouvert de tôles ondulées ou de planches, puis de matériaux rocheux et terreux et enfin par des «éclateurs » (plaques de béton armé protégeant les casemates des obus envoyés depuis les lignes allemandes). Toutefois, ces casemates enterrées étaient de dimensions plus grandes (jusqu'à 30 à $40 \mathrm{~m}$ ) que les fosses rectangulaires de la forêt de Grémecey qui n'ont probablement pas contenus de tels édifices. Ces fosses devaient plutôt abriter des abris temporaires en bois pour les hommes, les chevaux ou le matériel.

Quant aux dépressions aux contours rectangulaires situées en bordures du plateau, il semble qu'on puisse les mettre en rapport avec les « écuries souterraines " et les parcs à chevaux évoqués par la mémoire collective, d'autant plus que ces vestiges se situent à proximité de la fontaine Vassieux, source issue de l'aquifère du Calcaire à gryphées améliorée par des captages. Cependant, il ne reste aucun indice permettant d'affirmer, comme le prétend le récit local, que la fontaine se déversait dans un bassin situé en aval dans le chenal d'écoulement du vallon Vassieux. Un «abreuvoir bétonné » situé dans le vallon aurait permis aux hommes de disposer d'eau le long des tranchées d'approches vers la ligne de front au sud.

\section{c. Le ravitaillement de la position militaire de la forêt de Grémecey}

Dans le massif forestier de Grémecey, le paysage militaire de la Grande Guerre est resté dans un état de " congélation historique » partiel malgré le travail de comblement des tranchées et des abris. Les cicatrices ou plutôt les scarifications (casemates, tranchées, abris souterrains probables) (Nancy, 2013) sont nombreuses malgré une reconstitution d'un couvert forestier dense. Le fond du vallon conduit à une butte qui portait avant 1914, la ferme de Merlinsol, incendiée lors des escarmouches qui précèdent la bataille de Morhange. Cette position est devenue dès l'automne 1914 un poste avancé de la deuxième ligne allemande, en retrait par rapport à la première ligne située le long de la Seille. En outre, la position allemande de la forêt de Grémecey offrait une vue sur les positions françaises face aux lignes allemandes de la forêt de Bezange. 
Les nombreuses casemates situées sur le plateau ainsi que l'important réseau de lignes téléphoniques soulignent d'une part la profondeur du dispositif d'accès, et d'autre part l'importance stratégique du dispositif militaire. Pendant l'automne 1918, ces vestiges, en particulier ceux du vallon Vassieux, s'inscrivaient dans la zone avancée allemande, et plus précisément entre les zones d'arrêt et de résistance (doc. 7 et 8) situées sous couvert forestier. Les positions de combat (zone de surveillance) au sud du vallon occupent les versants de la vallée de la Seille partiellement contrôlée par les forces allemandes. En retrait sur le plateau, les positions de vie (casemates-abris, blockhaus de commandement, écuries, parcs à chevaux, positions d'artillerie) complètent le dispositif. En bordure d'un chemin forestier menant vers les villages de l'arrière, un petit monument rappelle un ancien cimetière militaire allemand. Plus au nord, le village de Fresnes-en Saulnois abritait, quant à lui, un poste médical et formait une zone de repos à l'arrière du front.

Document 7 : Modèle du polémo-paysage de la Forêt de Grémecey et du vallon Vassieux pendant la Grande guerre

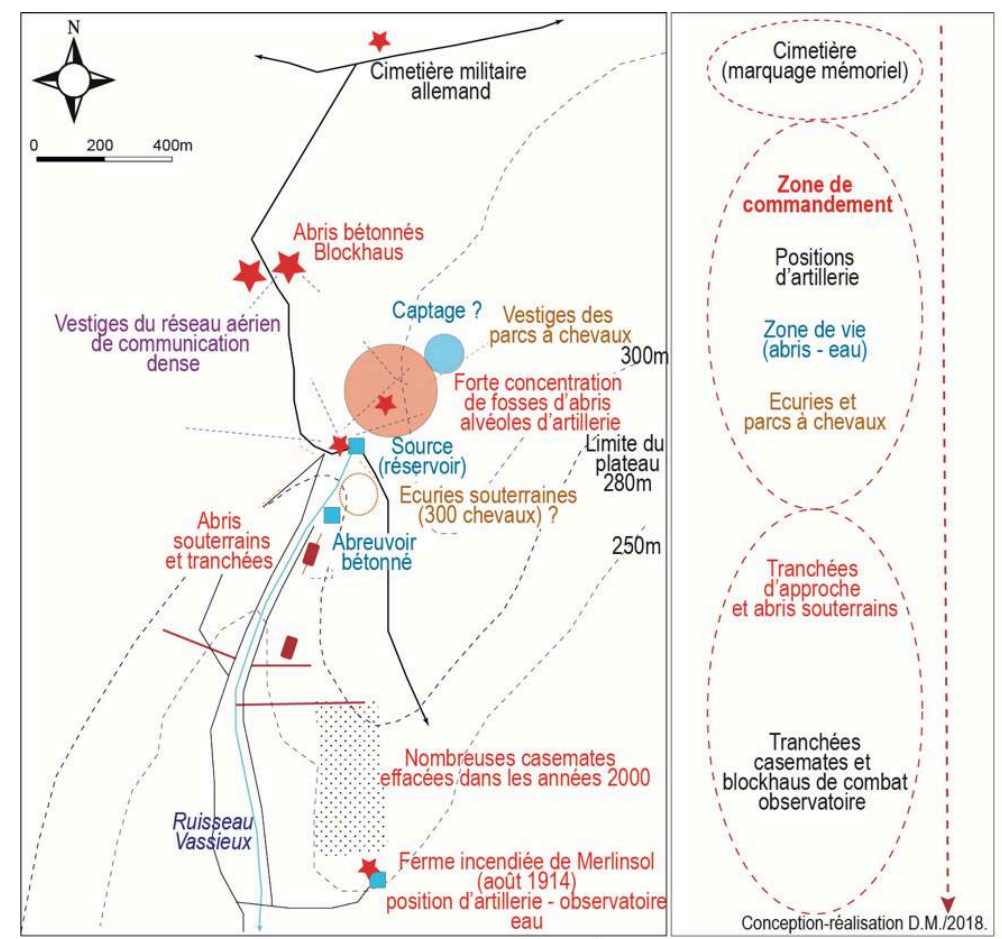

Fig. 17 : Modèle du polémo-paysage de la Forêt de Grémecey et du vallon Vassieux pendant la Grande Guerre 
Document 8 : Carte des positions allemandes en Lorraine centrale en septembre 1918

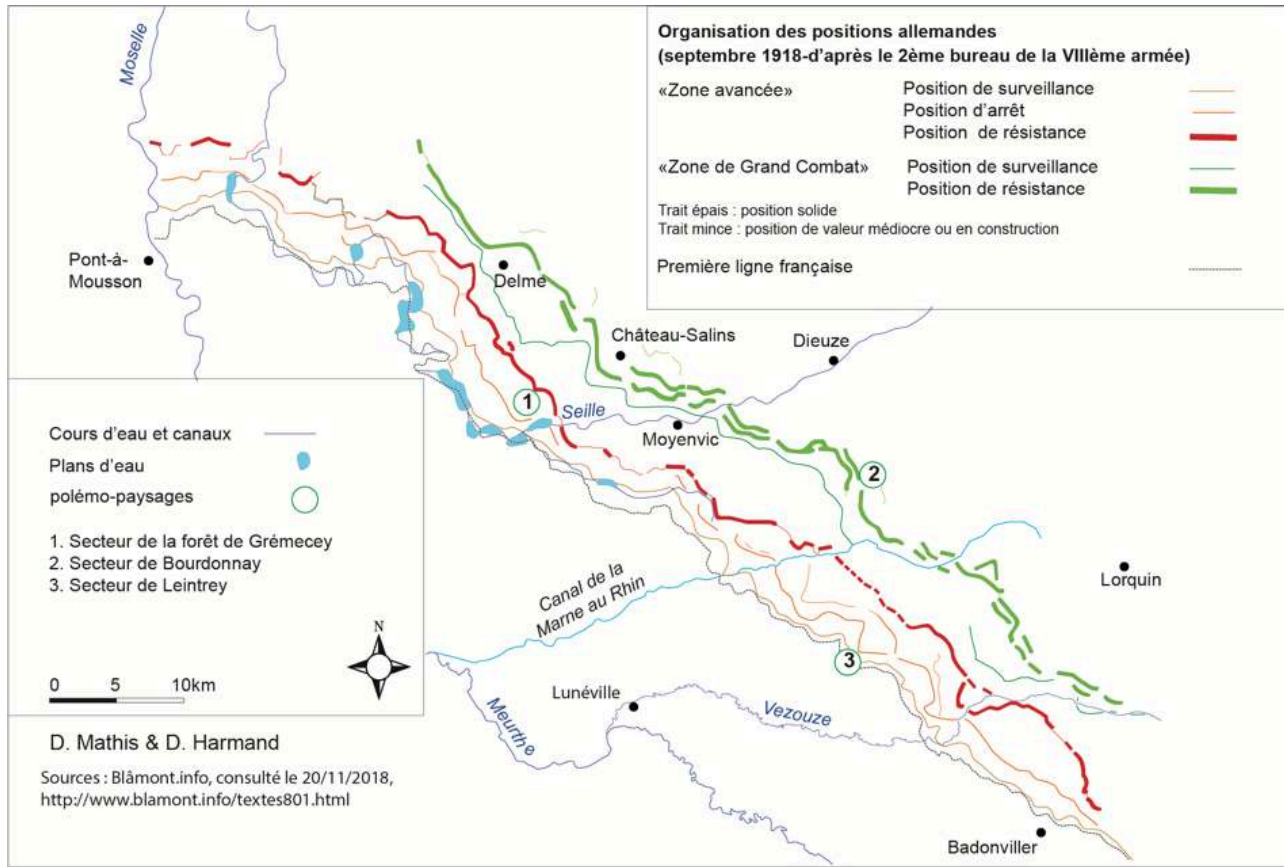

Fig. 18 : Carte des positions allemandes en Lorraine centrale en septembre 1918

'édification de ce dispositif de défense et son ravitaillement impliquent l'existence de voies de communication apportant notamment matériel lourd et matériaux. La mémoire collective a conservé le souvenir d'une voie ferrée à voie étroite reliant sur le plateau la gare de Fresnes-en Saulnois qui était située à l'est du village et la forêt de Grémecey. Cette localité était, quant à elle, reliée par la voie ferrée jusqu'à Metz. C'est par voie ferrée qu'ont été acheminés les tonnages élevés de matériaux utilisés pour construire les nombreux blockhaus observés. Leurs murs contiennent des galets parfaitement arrondis de dimensions centimétriques (jusqu'à $8 \mathrm{~cm}$ ) qui trahissent leur origine. Ces galets de quartz et de quartzite sont issus du Conglomérat principal du Buntsandstein (Trias). Toutefois, il est peu probable que ces éléments aient été extraits d'une carrière entaillant le substratum triasique des Vosges gréseuses ou du Warndt, ne serait-ce que parce que ces galets sont liés par un ciment siliceux très résistant. Il est beaucoup plus probable que les matériaux aient été extraits dans des gravières de fond de vallée ou des terrasses alluviales du bassin de la Moselle (vallées de la Meurthe et de ses affluents, Vezouze et Mortagne, de la Sarre et terrasses de la Moselle antérieure à la capture de celle-ci par le réseau du Rhin - et alimentées par la Paléo-Meurthe (Cordier et al.,2004; Harmand et Cordier, 2012). Il est possible que cette dernière source d'approvisionnement ait fourni les galets siliceux des blockhaus du secteur étudié, car il existe au nord de Metz, en rive gauche de la Moselle, de vastes terrasses alluviales ante-capture exploitées en carrières et reliées au réseau ferré.

Quant à l'approvisionnement en eau du cantonnement de la forêt de Grémecey, il est douteux que la fontaine Vassieux ait été suffisante eu égard à la modestie du débit des sources de l'aquifère des Calcaires à gryphées. Soit l'approvisionnement en eau s'effectuait à partir de l'aquifère du Rhétien et notamment des sources du vallon du ruisseau de Blanche Fontaine, situé à l'ouest du vallon Vassieux, soit une partie de l'approvisionnement était acheminépar voie ferrée.

Revue de géographie historique, 14-15 | 2019 


\section{Vestiges et espaces mémoriaux de la Grande guerre en Lorraine centrale de la côte de la Seille aux Vosges gréseuses (exclues)}

\section{A. Les espaces mémoriaux de la Lorraine centrale}

Dans une dernière partie, il est nécessaire de replacer le polémo-paysage de la forêt de Grémecey dans les espaces mémoriels de la Lorraine centrale entre la vallée de la Seille et le Grand Couronné d'une part et les Vosges gréseuses de l'autre. La question est en effet pertinente dans la mesure où la forêt de Grémecey se trouve sur un front resté à peu près stable pendant les quatre années du conflit et qui coïncide avec la frontière entre la France et l'Empire allemand. Le secteur étudié se trouve aussi à la charnière d'un front qui, vers l'est, s'est déplacé sur plusieurs dizaines de kilomètres à la fin de l'été 1914, vers le nord lors de l'offensive française de Morhange, et vers le sud et le sud-ouest au cours des offensives allemandes de la Trouée de Charmes et du Grand Couronné avant de se stabiliser légèrement au sud de l'ancienne frontière francoallemande à l'automne 1914.

31 Sur cet espace du plateau lorrain compris entre la Nied française au nord et la Moselle au sud, les vestiges de la Grande guerre s'organisent en trois lignes paysagères (Doc 9) : 1) La ligne de la bataille de Morhange au nord couvre le Plateau lorrain, lescôtes de Saffais et la dépression marneuse du Keuper (bassin de la Petite-Seille (Doc. 1). Les traces des combats sont limitées. Elles concernent les villages détruits-reconstruits. Les marqueurs mémoriels concernent essentiellement les cimetières (10 nécropoles nationales et 5 monuments commémoratifs, ainsi que 8 carrés militaires dans d'autres cimetières (Didier, 2007). Le « paysage de la mort, «matérialisé par les petits cimetières au contact du front a été, dans cette ligne paysagère comme dans les deux autres, transformé par le regroupement des tombes vers les grandes nécropoles. Ainsi, dès 1923, le cimetière allemand de l'Hellenwald à Morhange regroupe les tombes de soldats allemands issus de 51 communes.

2) La ligne des combats du Grand Couronné prolongée par la Trouée de Charmes concerne d'abord le secteur situé au nord-est de Nancyoùles traces des combats sont également limitées aux villages détruits (Réméréville, Champenoux). Trois nécropoles ponctuent le plateau des Calcaires à gryphées marqué par 10 monuments commémoratifs (dont Courbesseauxoùse trouve le monument dédié au Général Castelnau). Les autres espaces mémoriels concernent ensuite le fort de Manonviller et plusieurs monuments commémoratifs. Le fort de Manonviller bombardé (25-27 août), puis dynamité le 12 septembre - parce que les tourelles étaient inutilisables après les bombardements -, constitue un des éléments marquants des combats. Toutefois, actuellement, ce fort d'arrêt de la ligne Séré de Rivières, géré par une Association loi 1901, n'est ouvert qu'exceptionnellement au public et fait encore l'objet de travaux de restauration. Les principaux monuments commémoratifs se trouvent en premier lieu sur la butte-témoin du Léomont, situé comme le fort de Manonviller sur les reliefs de la côte éponyme. Le monument du Léomont $(336 \mathrm{~m})$, lieu de la résistance des troupes françaises (Division de fer) constitue un géosymbole du paysage de la bataille du Grand Couronné. Les deux autres monuments commémoratifs sont ceux de la Trouée de Charmes et du Grand Couronné. Le premier, qui domine la ville de Charmes, est situé au sommet de la côte des Calcaires à gryphées, en rive gauche de la Moselle. Le second se 
trouve sur la butte-témoin de Sainte-Geneviève, située comme les autres buttestémoins du Grand Couronné, en avant de la côte de Moselle (Harmand et al.,2018).

3) Entre ces deux lignes de bataille qui marquent les « hautes eaux » des progressions françaises puis allemandes, le front s'est stabilisé en dépit des offensives des deux camps. Dans cet espace localisé essentiellement dans le département de Meurthe-et Moselle existent de nombreux espaces mémoriaux valorisés par le conseil départemental (Facq (dir.), 2013). Outre la forêt de Ranzey, l'espace muséal Chaubet à Vitrimont, citons l'hôpital militaire souterrain de Domjevin, l'abri bétonné du Kronprinz à Emberménil ou les entonnoirs de Leintrey, haut lieu de la guerre des mines en Lorraine.

\section{Document 9 : Les principales nécropoles françaises et allemandes de la Grande guerre en Lorraine centrale}

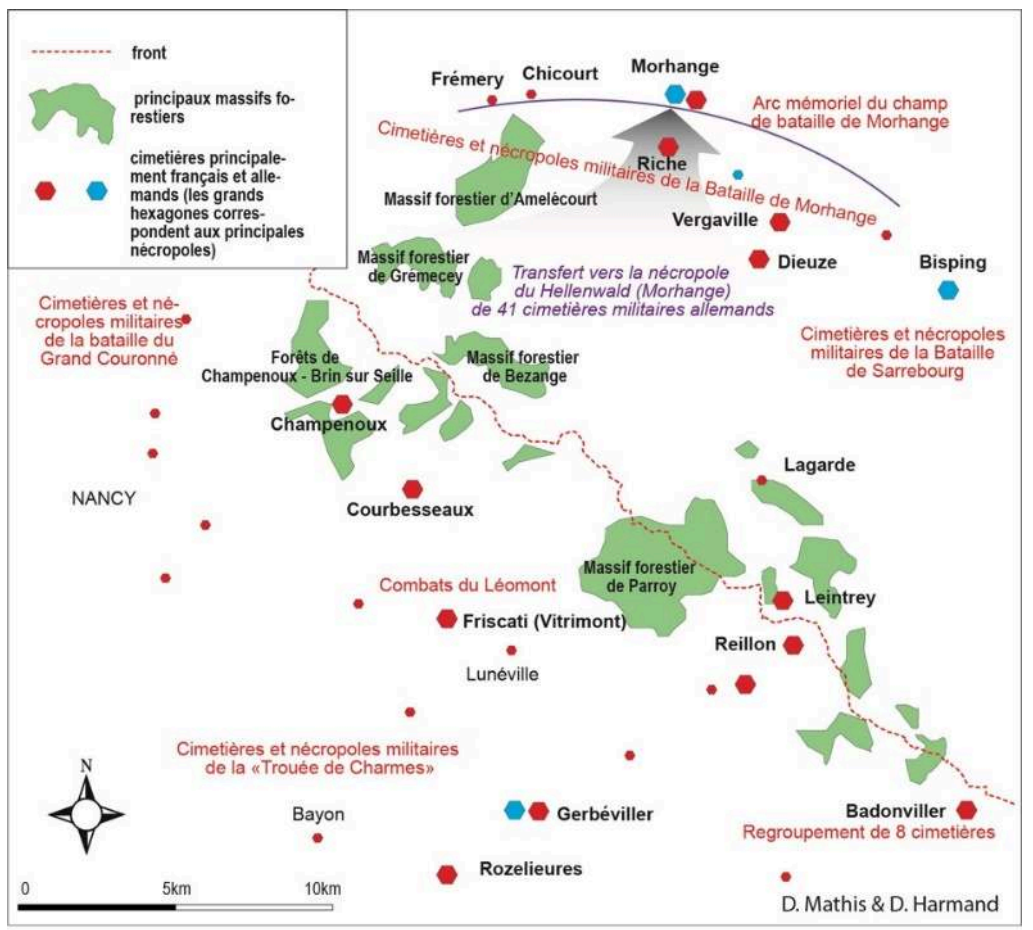

Fig. 19 : Carte des principales nécropoles françaises et allemandes de la Grande guerre en Lorraine centrale

C'est toutefois en forêt que le paysage de la guerre de la position est plus marqué : forêts de Brin-sur-Seille et d'Amance, de Bézange-la-Grande et de Parroy où les vestiges de la Grande Guerre, nombreux, ne sont pas signalés sur les cartes topographiques de l'IGN et sont valorisés par des associations locales.

On retrouve le long de la ligne de front de nombreux villages reconstruits. Pour la Meurthe et Moselle, les reconstructions sont en style «régionaliste». En Moselle, les reconstructions ont un style moins affirmé. De nombreux ouvrages fortifiés (casemates, blockhaus) sont restés en l'état dans les villages (comme dans la rue haute de Brin-surSeille) et au cœur des espaces agricoles. 


\section{B. L'inscription sur la liste de l'Unesco}

En 2011, un projet de classement au patrimoine de l'Unesco des « paysages de guerre du front occidental de la Grande Guerre » a été déposé (Unesco, année inconnue). Il a été requalifié en proposition de classement des nécropoles et sites mémoriels de la Première Guerre mondiale (front Ouest). Toutefois, le projet de classement a été reporté à 2021. Les cent cinq sites retenus le long des $700 \mathrm{~km}$ du front occidental se déclinent en nécropoles, monuments, stèles, chapelles commémoratives et mémoriaux. Ils témoignent de la violence du conflit mondial qui marque une rupture majeure dans l'histoire de l'humanité, traduisent une nouvelle approche du culte des soldats morts au combat et rappellent l'importance des pertes humaines.

Document 10 : Photographie de la nécropole allemande du Hellenwald à Morhange (cl. D. Mathis).

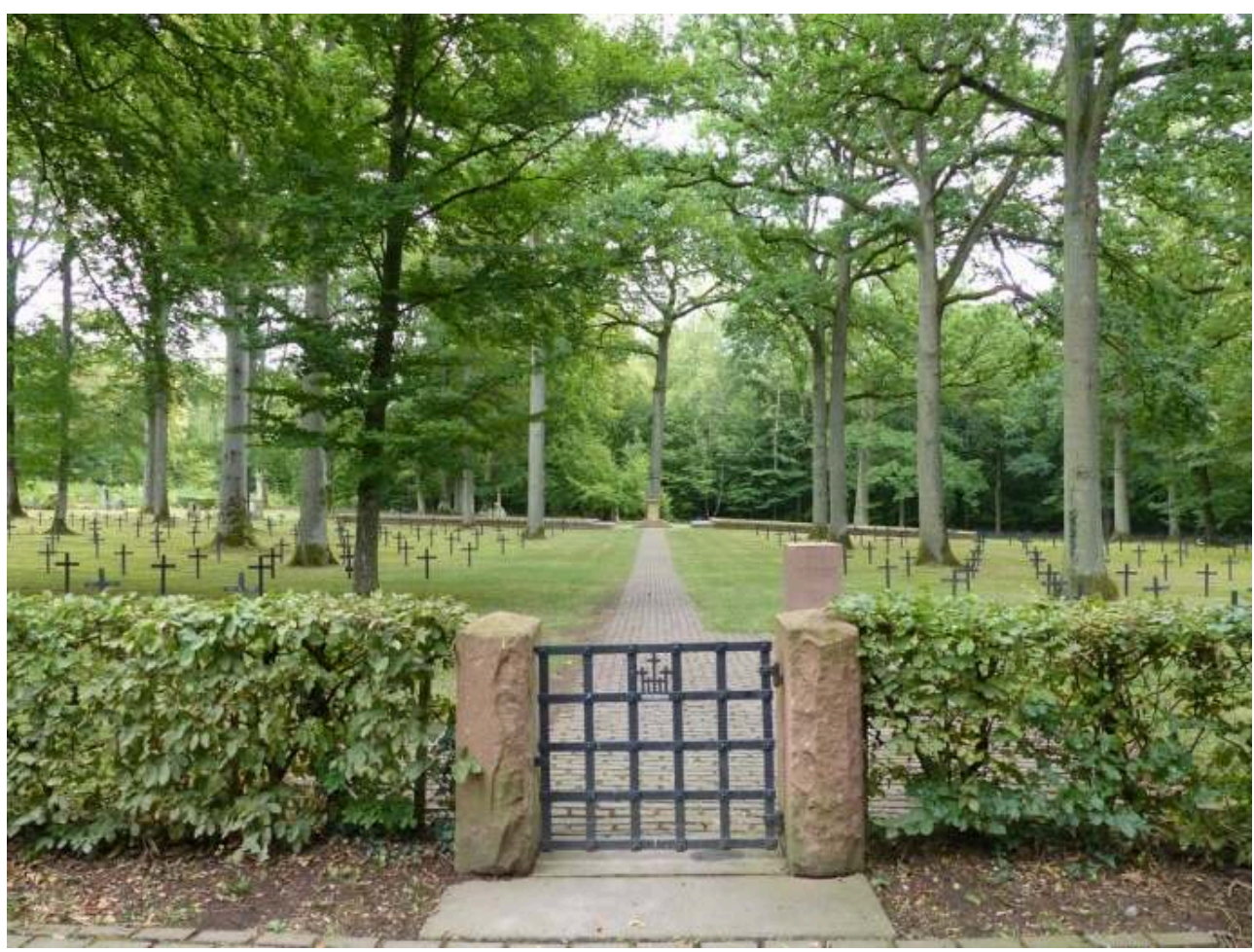

Il est nécessaire de préciser que pendant les deux premiers mois de la Grande Guerre, lors des violents combats des batailles de Morhange, du Grand Couronné et de la Trouée de Charmes, les pertes ont été considérables : 17000 morts pour l'armée allemande (Bavarois) et plus de 60000 blessés, disparus et prisonniers, et environ autant pour l'armée française (Roth, 2014). Les quinze sites retenus pour le département de la Moselle avec les nécropoles et les monuments de Frémery, de Chicourt, de Conthil, de Riche, ou de Morhange témoignent des combats de 1914 et d'un territoire de guerre où la mémoire autour de la bataille de Morhange a été plus difficile à construire. La nécropole française de Vitrimont-Friscati et le monument du Léomont associés aux batailles du Grand Couronné et de la Trouée de Charmes, sont les sites retenus en Meurthe-et-Moselle. 


\section{La mémoire souvent défaillante en Moselle}

En dehors des nécropoles et des monuments commémoratifs, les autres vestiges situés dans le département de la Moselle - et par conséquent dans l'Empire allemand jusqu'en 1918 -, sont, comme ceux de la forêt de Grémecey, non entretenus par la mémoire collective. Il est probable que d'autres massifs forestiers renferment d'autres traces. Les exemples les plus significatifs concernent les 3 sites d'artilleries lourdes de Hampont/ Morville-lès-Vic, de la forêt de Grémecey et de Burthecourt. Le plus célèbre d'entre eux est celui du «Gros Max », canon Krupp d'infanterie de marine de $38 \mathrm{~cm}$ et de $260 \mathrm{t}$, qui, en 1916 et 1917, bombarda Nancy situé à 31 km, Dombasle et Lunéville (Morville-lèsVic, 2011). Le site, localisé à l'est de Château-Salins, à l'extrémité ouest de la buttetémoin du Calcaire à gryphées de la Forêt de Bride, légèrement en contrebas au nord de la corniche sommitale, est au cœur d'un polémo-paysage actuellement sous forêt et difficilement accessible. Ce dernier est constitué notamment par la cuve de lancement $\mathrm{du}$ canon et de 2 galeries de $200 \mathrm{~m}$ de longueur. L'emplacement de la pièce d'artillerie lourde de la forêt de Grémecey, est davantage accessible, puisque situé en bordure d'un chemin forestier, mais le site n'est pas valorisé. En outre, le diamètre du canon est inconnu, tout comme ses objectifs à atteindre. Quant à la pièce d'artillerie lourde de Burthécourt, village situé entre Chambrey et Château-Salins, nul ne sait où elle se trouvait.

Dans les zones de culture, les traces de la Grande Guerre sont davantage visibles certains secteurs du front ont conservé une densité élevée de blockhaus, comme à La Bourdonnay. Toutefois, de nombreux blockhaus de la Lorraine centrale ont été enterrés, comme ce fut le cas dans les années 2000 sur la côte de la Seille au sud-est du vallon Vassieux (Fig. 1)

\section{Conclusion}

Peu connus, les polémo-paysages de la Lorraine centrale sont associés à la vaste dépression marneuse du Keuper et à un relief de côtes plus discret que dans la Lorraine occidentale. Toutefois, le relief des côtes de Saffais qui se décline en côte de la Seille (des Calcaires à gryphées), localement de Port (des grès rhétiens) et du Léomont (de la Dolomie de Beaumont) ont joué un rôle majeur lors des batailles de Morhange, de la Trouée de Charmes et du Grand Couronné d'août à septembre 1914. Dans cette partie centrale de la Lorraine qui, en France, est entrée la première dans la Grande guerre, les vestiges des polémo-paysages sont nombreux et complexes sous forêt, éparses, isolés et fossilisés dans les espaces ouverts, dans les villages reconstruits, si bien qu'en changeant d'échelle, le paysage hérité de la guerre de position est discontinu, en peau de panthère.

41 D'un point de vue temporel, comme souvent pour les «fronts » aménagés et les lignes de défense, la fossilisation des traces, leur "congélation historique " sous le couvert forestier, n'est pas exceptionnelle, elle s'observe dans le cas de la plupart des grandes fortifications de campagne et lignes défensives constituées au XVIII ${ }^{e}$ siècle (Mathis, 2016). C'est en effet au sein des différents massifs forestiers qui ponctuent l'ancienne ligne de front, en miroir de part et d'autre des positions françaises et allemandes que se trouvent les vestiges les plus nombreux de la Grande Guerre. Il est possible de hiérarchiser les espaces mémoriels en trois types: 1) les nécropoles et monuments 
commémoratifs majeurs en partie inscrits sur la liste indicative de l'Unesco au titre des nécropoles et sites mémoriels du front occidental de la Première Guerre mondiale ; 2) les vestiges aménagés par les pouvoirs publics ou les associations et qui font l'objet d'un tourisme mémoriel au moins partiel ; 3) les vestiges plus ou moins oubliés, visibles ou invisibles, qui pour la plupart se trouvent dans le département de la Moselle.

\section{BIBLIOGRAPHIE}

Bergerat F. (Dir.), 2018, 14-18, La Terre et le Feu. Géologie et géologues sur le front occidental, Paris, coédition AGBP-COFRHIGÉO-SGN, $450 \mathrm{p}$.

Boulanger P., 2002, La géographie militaire (1871-1939), Paris, Economica, 619 p.

Brin-sur-Seille (1914-1918), 2017, Les casemates de la forêt d'Amance, consulté en 2019, URL : https:// brin1418.wordpress.com/2017/01/14/les-casemates-de-la-foret-damance

Cochet F., 2014, « Les entrées en guerre et les premières batailles sur le sol lorrain » In LaborieBarrière L., 2014, Été 1914. Nancy et la Lorraine dans la guerre, Serge Domini (éd.), pp. 76-85.

Conseil Départemental de Meurthe-et- Moselle, année inconnue, La forêt de Ranzey, consulté en 2019, URL : http:// www.citedespaysages.meurthe-et-moselle.fr/les-espaces-naturels-sensibles/ autour-de-lunéville/la-forêt-de-ranzey

Cordier S., Harmand D., Losson B. et Beiner M., 2004, « Alluviation in the Meurthe and Moselle valleys (Eastern Paris Basin, France): lithological contribution to the study of the Moselle capture and Pleistocene climatic fluctuations», Quaternaire, 15, (1-2), pp. 65-76.

Deshaies M., 1999, «L'explication du relief de côte de la France dans la première moitié du XXème siècle ", Revue Géographique de l'Est, 39, pp. 21-30.

Didier J., 2007, Guide des lieux de mémoire. Morhange, le Grand Couronné de Nancy, Ysec, 152 p.

Durand M., 2014, « Le Trias de l'Est du Bassin parisien. » In. : Gély J.-P. et Hanot F. (dir), 2014, Le Bassin parisien, un nouveau regard sur la géologie. Cinquantenaire de l'Association des Géologues du Bassin de Paris, pp. 50-57.

Facq V. (dir), 2013, La Grande Guerre en Meurthe-et-Moselle : 1914-1918, Meurthe \& Moselle Tourisme, consulté en 2019, URL : http://centenaire.org/sites/default/files/ references-files/edition-ducentenaire- 2013-web_0.pdf

Gély J.-P. et Hanot F. (dir), 2014, Le Bassin parisien, un nouveau regard sur la géologie. Cinquantenaire de l'Association des Géologues du Bassin de Paris, 229 p. + 1 pl. h. t.

Harmand D. et Cordier S., 2012, « The Pleistocene terrace staircases of the present and past rivers downstream from the Vosges Massif (Meuse and Moselle catchments). », Netherlands Journal of geosciences-Geologie en Mijnbouw, 91- 1/2, pp. 91-109.

Harmand D., Porchier J.-C., Le Roux J., Harmand G., Chiffre E., 2018. « Les batailles de Morhange, de la Trouée de Charmes et du Grand Couronné de Nancy. » In. : Bergerat F. (dir), 14-18, La Terre et le Feu. Géologie et géologues sur le front occidental, Paris, co-édition AGBP-COFRHIGÉO-SGN, 450 p. 
Le Roux J., 1978, « Terrains secondaires et structure ", In. : Notice explicative, et Carte géol. France (1/50 000), feuille NANCY (230), Orléans, Bureau de recherches géologiques et minières.

Le Roux J., 1999, « Le contexte structural de l'est du Bassin parisien et les relations avec la sédimentation. », Bull. Inf. Geol. Bass. Paris, vol. 36, N 1, pp. 7-13.

Le Roux J. et Harmand D., 2014, « Le relief de côte de l'Est du Bassin parisien. » In.: Gély J.-P. et Hanot F. (dir), Le Bassin parisien, un nouveau regard sur la géologie. Cinquantenaire de l'Association des Géologues du Bassin de Paris. 92 points remarquables le long du tracé de la coupe géologique, point $n^{\circ} 22$, p. 147.

Le Roux J. et Harmand D., 2015, « Les enseignements du relief de côtes dans l'est du Bassin parisien. », Bulletin Inf. Géol. Bass. Paris, Vol. 51, pp. 24-39.

Liedtke H, Deshaies M., Gamez P., Harmand D., Preusser H., 2010, Die Oberflächenformen in der Grenzregion Saarland - Lothringen - Luxembourg. / Les formes de relief dans la région frontalière SarreLorraine-Luxembourg. Veröffentlichungen des Instituts für Landeskunde im Saarland. Saarbrücken, Band 48. / Forschungen zur deutschen Landeskunde. Liepzig, Band 259, 367 p + 1 carte h. t. (carte à 1/300 000 de l'espace Saar-Lor-Lux). [Ouvrage collectif bilingue francoallemand].

Mathis D., 2016, « Les hydrosystèmes militaires défensifs de Basse-Alsace (XVIIe-XVIIIe siècles) ", Revue de Géographie Historique, $\mathrm{n}^{\circ} 8$, Géographie historique et questions militaires (1), URL : http://rgh.univ-lorraine.fr/articles/ view/70/

Morville-lès-Vic, 2011, Le Gros Max à Morville-lès-Vic, consulté en 2019, URL : http://www.morvilleles-vic.com/le-gros-max-a-morville-les-vic.php

Nancy J.-L., 2013, « Paysage avec cicatrices », In. : Les cicatrices du paysage, Les cahiers de l'École de Blois, n¹1, Éditions de la Villette, pp. 10-17.

Roth F., 2014, Six mois qui incendièrent le monde. Juillet-decembre1914. Éd. Tallandier, 552 p.

Schmitthenner H., 1923, Die Oberflächenformen der Stufenlandschaft zwischen Maas und Mosel. Verlag von j. Engelhorn nachf., Stuttgart, $89 \mathrm{~S}$.

Taborelli P., Devos A., Desfossés Y., Brénot J., Laratte S., 2017, « Typologie et organisation spatiale des « polémo-formes » de la Grande Guerre révélées par l'outil LiDAR et les Plans directeurs. Application à la Champagne et à l'Argonne. ", Géographie historique et guerres, N10- 11 mai 2017.

Unesco, année inconnue, Sites funéraires et mémoriels de la Première Guerre mondiale (Front Ouest) (France), consulté en 2019, URL : https://whc.unesco. org/fr/listesindicatives/5884/

Villate R., 1925, Les conditions géographiques de la guerre. Étude de géographie militaire sur le Front Français de 1914 à 1918. Paris, Éd. Payot, 350 p. 54

\section{RÉSUMÉS}

La Lorraine centrale présente successivement d'ouest en est, 1) la côte double de Saffais constituée au sommet par la côte de la Seille (ou du Calcaire à gryphées de l'HettangienSinémurien) ou la côte de Saint-Nicolas-de-Port (ou des grès rhétiens) et àla basepar la côte du Léomont (ou de la Dolomie de Beaumont, Keuper), 2) la vaste dépression marneuse du Keuper, et 3) la côte de Lorraine (ou du Muschelkalk). C'est dans cette région à la structure complexe de dômes et de cuvettes dans laquelle s'inscrit un relief de côte peu élevé, découpé et précédé par de nombreuses buttes-témoins, que se sont déroulées en août et septembre 1914 les batailles très meurtrières de Morhange, de la Trouée de Charmes et du Grand Couronné de Nancy. C'est ici que 
le front se fixa, à partir de la fin 1914 jusqu'en 1918, légèrement au sud du tracé de la frontière franco-allemande. L'objet de cette recherche a consisté à mettre en évidence les nombreux vestiges de la Grande guerre, conservés dans la forêt de Grémecey (Moselle) située sur la marge sud-ouest de l'Empire allemand. Ces derniers, situés sur le plateau des Calcaires à gryphées(nombreux blockhaus à demi enterrés, dépressions aux contours géométriques) et dans le vallon de Vassieux qui entaille la côte de la Seille (tranchées sinueuses dans les marnes du Keuper ou abris souterrains dans les grès rhétiens) et reliés par plusieurs lignes téléphoniques, ont permis de reconstituer un centre de commandement organisé en fonction du relief de côte. D'autres vestiges notables existent en Lorraine centrale. Ils participent à la mise en valeur patrimoniale, surtout dans le département de la Meurthe-et-Moselle (Léomont, entonnoirs de mines de Leintrey), mais assez peu dans le département de la Moselle où existent pourtant des sites majeurs, comme les emplacements des pièces d'artillerie lourde de marine de Hampont ou de la Forêt de Grémecey. Cependant, les principaux espaces mémoriels de la Première guerre mondiale de la Lorraine centrale sont les nécropoles à la fois françaises et allemandes, nées après le regroupement des sépultures après la Grande guerre et dont certaines sont inscrites sur la liste indicative de l'Unesco.

The central Lorraine presents successively from west to east, 1) the scarpland of Saffais, formed at the top by the Seille (or of the «Hettangian-Sinemurian Calcaires à gryphées » formation) cuesta or the Saint-Nicolas-de-Port (or Rhetian sandstones) cuesta and at the base by the Leomont (or the Keuper « Dolomie de Beaumont " formation) cuesta, 2) the large depression of Keuper marls, and 3) the Lorraine (or Muschelkalk) cuesta. From the end of 1914 until 1918, the front line settled, slightly in the South of the French-German border.In this low eroded scarpland preceded by the numerous outliers and carved in the complexe domes and syncline structure took place in August and September, 1914 the very deadly battles of Morhange, the "Trouée de Charmes » and the "GrandCouronné of Nancy ». The object of this research was to show the many vestiges of the Great War, preserved in the Forest of Grémecey (Moselle) located on the south-western margin of the German Empire. The latter, located on the plateau of the Liasic "Calcaire à gryphées » (many half-buried blockhouses, depressions with geometric outlines) and inthe Vassieux small valley intrenched in the Seille cuesta (sinuous military trenches on the Keuper marls or underground shelters in the Rhetian sandstones) connected by several telephone lines allowed to reconstruct a command center organized according to the relief of scarpland.Other notable remains of the Great War exist in central Lorraine. They participate in the heritage enhancement especially in the department of Meurthe-et-Moselle (Léomont, mine craters of Leintrey), but less in the Moselle department where there are nevertheless major sites, such as the locations of parts heavy marine guns from Hampont or the Gremecey Forest. However, the main memory areas of the Great War of Central Lorraine are the great both French and German cemeteries, etablished after the regrouping of graves after the Great War and some of which are listed on the indicative list of Unesco.

\section{INDEX}

Keywords : scarpland of Saffais, of the Seille, of Saint-Nicolas-de-Port, of the Léomont, Great War, polemo-landscapes, cemeteries, Grémecey, Vassieux

Mots-clés : Côtes de Saffais, de la Seille, de (Saint-Nicolas-de-) Port, du Léomont, Grande Guerre, polémo-paysages, nécropoles, forêt de Grémecey, Vassieux 


\section{AUTEURS}

\section{EMMANUEL CHIFFRE}

Laboratoire Loterr, Université de Lorraine 